\title{
Travel Distance Does Not Affect Outcomes After Arthroscopic Rotator Cuff Repair
}

\author{
Nabil Mehta, M.D., Ophelie Z. Lavoie-Gagne, B.S., Matthew R. Cohn, M.D., \\ Joseph Michalski, M.P.H, M.S., Ashlyn Fitch, B.S., Adam B. Yanke, M.D. Ph.D., \\ Brian J. Cole, M.D., M.B.A., Nikhil N. Verma, M.D., and Brian Forsythe, M.D.
}

\begin{abstract}
Purpose: To determine the effect of travel distance on achieving the minimal clinically important difference (MCID) on patient-reported outcome measures (PROMs) at least 1 year after arthroscopic rotator cuff repair (RCR). Methods: Patients undergoing primary arthroscopic RCR with a minimum l-year follow-up at a high-volume tertiary referral center between May 2017 and June 2019 were retrospectively reviewed. Patients were divided into two groups: driving distance of $>50$ miles from the institution (referral group) and $<50$ miles (local group). American Shoulder and Elbow Score (ASES), Single Assessment Numeric Evaluation (SANE) score and Constant score at minimum 1-year followup were assessed. Chi-square analysis was used to analyze achievement of MCID on any PROM. Subgroup analysis by tear complexity ( 1 tendon vs. $>2$ tendons torn) was conducted. Logistic regression was performed to determine whether travel distance and other variables of interest had an effect on achieving MCID. Results: Of 996 patients reviewed, 385 patients $(38.7 \%)$ had minimum 1 -year follow-up. Of these, $62(16 \%)$ traveled $>50$ miles to their orthopedic provider, and 212 $(55 \%)$ had a complex tear. MCID for at least one PROM was reached by 305 (94\%) patients in the local group and 56 $(90 \%)$ patients in the referral group. There was no significant difference between groups in reaching MCID for any PROM. Subgroup analysis by tear complexity revealed no significant difference in preoperative PROMs or achieving MCID postoperatively between referral groups. There was no significant difference between groups when controlling for age, sex, adjusted gross income (AGI), primary health insurance, tear chronicity, and tear complexity. Conclusions: When controlling for age, sex, AGI, primary health insurance, tear chronicity, and tear complexity, distance to a high-volume shoulder arthroscopy surgery center did not have an effect on achieving the MCID for any PROM at least 1 year after arthroscopic RCR. Level of Evidence: Level III, retrospective cohort study.
\end{abstract}

\section{Introduction}

A s health care continues to shift to value-based models, many patients are seeking care at highvolume specialty centers. Streamlined clinical care pathways, higher-volume providers, and potentially

Department of Orthopaedic Surgery, Rush University Medical Center, Chicago, Illinois, U.S.A.

The authors report the following potential conflicts of interest or sources of funding: B.F. reports being a paid consultant for Stryker and Jace Medical; he has received research support from Arthrex, Smith $\theta$ Nephew, and Stryker. N.V. reports being a paid consultant for Minivasive and Orthospace; he has board membership in the American Orthopaedic Society for Sports Medicine, the American Should and Elbow Surgeons, the Arthroscopy Association of North America, Knee, and SLACK, Inc.; he has received research support from Arthrex, Breg, Ossur, Smith $\theta$ Nephew, Wright Medical Technology; he has received royalties from Arthroscopy, Smith $\theta$ Nephew, Vindico MedicalOrthopedics Hyperguide; and he has stock or stock options in Cymedica, Minivasive, and Omeros. B.C. reports board membership in the American Journal of Orthopaedics, American Journal of Sports Medicine, Cartilage, International Cartilage Repair Society, Journal of Shoulder and Elbow Surgery, Journal of the American Academy of Orthopaedics Surgeons; he is a paid consultant for Arthrex, Aesculap, B. Braun, the lower costs make high-volume regional centers of Centers of Excellence (COEs) an appealing option for patients. ${ }^{1,2}$ A number of previous studies have suggested that higher-volume surgeons often produce improved patient outcomes; lower complication rates

National Institutes of Health (NIAMS $\theta$ NICHD), and Regentis; and he has received royalties from Elsevier Publishing and Operative Techniques in Sports Medicine. A.Y. reports being a paid consultant for ConMed Linvatec, JRF Ortho, and Olympus; he has received research support from Arthrex, Organogenesis, and Vericel; and he has stock options from Patient IQ. Full ICMJE author disclosure forms are available for this article online, as supplementary material.

Received November 10, 2020; accepted October 24, 2021.

Address correspondence to Nabil Mehta, M.D., Department of Orthopaedic Surgery, Rush University Medical Center, 1611 W. Harrison St., Suite 300, Chicago,IL 60612,U.S.A.E-mail: Mehta.nabil@gmail.com

(C) 2021 THE AUTHORS. Published by Elsevier Inc. on behalf of the Arthroscopy Association of North America. This is an open access article under the CC BY-NC-ND license (http://creativecommons.org/licenses/by-nc-nd/4.0/). 2666-061X/201795

https://doi.org/10.1016/j.asmr.2021.10.030 
have been observed among higher-volume surgeons performing total joint arthroplasty, hip arthroscopy, and various spine surgeries. ${ }^{2-6}$ Similarly, lengths of stay, operating room times, and reoperation rates were found to be higher among shoulder surgeons who performed less than 12 rotator cuff repairs per year. ${ }^{7}$

While patients may wish to establish care at COEs in hopes of achieving better outcomes, a significant travel distance may be required to access such centers. Patients who live far from their treating surgeon's practice may have difficulty accessing their provider if an adverse event occurs or may be unable to undergo rehabilitation within their provider's system. Indeed, a "reverse travel bias" has been described in the general surgery literature, in which patients who travel greater distances to their provider have higher risk of complications compared to patients treated locally. ${ }^{8}$ Recent studies in the orthopedic literature have shown that travel distance does not affect outcomes following total joint arthroplasty or hip arthroscopy, although this effect remains unknown after arthroscopic rotator cuff repair (RCR). ${ }^{9,10}$

Arthroscopic RCR is among the most common orthopedic procedures performed in the United States, with an incidence of 23.5 per 100,000 , and it is a common procedure at tertiary referral centers. ${ }^{11}$ The purpose of this investigation is to determine the effect of increasing travel distances on achieving the minimal clinically important difference (MCID) on patientreported outcome measures (PROMs) at least 1 year following arthroscopic rotator cuff repair. We hypothesized that a greater travel distance would not reduce MCID achievement or PROMs following RCR.

\section{Methods}

This study was approved by the Institutional Review Board, and no funding was received. Data were prospectively gathered on all patients undergoing RCR for the treatment of partial or full-thickness rotator cuff tears from May 2017 to June 2019 by 4 fellowshiptrained surgeons (A.B.Y., B.J.C., N.N.V., and B.F.). Inclusion criteria were patients who underwent primary RCR after failure of conservative management (physical therapy, activity modification, oral anti-inflammatories, and cortisone injections) with a minimum l-year follow-up. Exclusion criteria were patients undergoing revision rotator cuff repair, patients with isolated subscapularis tears, unavailable patient-reported outcome measures (PROMs) at a minimum of 1 year postoperatively, unavailable geographic demographic information (postal code at the time of surgery), or having a residence further than the bordering states of the institution of study (Illinois, Indiana, and Michigan) that would have likely required transportation outside of ground transport.

\section{Surgical Technique}

RCR was performed by one of the four senior authors (A.B.Y., B.J.C., N.N.V., and B.F.) via standard posterior, anterior, and lateral portals using their preferred technique, as previously described. ${ }^{12-14}$ All surgeons were fellowship-trained surgeons in sports medicine practicing at a high-volume orthopaedic surgery practice. All perform a high volume of RCRs, and all are within the top $1 \%$ of work relative value units by Medical Group Management Association data.

\section{Postoperative Rehabilitation}

All patients underwent the standard postoperative rehabilitation protocol practiced at the institution of study. In brief, patients were discharged on the day of the surgery and immobilized in a sling for 6 weeks. After the first postoperative visit, typically 1 to 2 weeks after surgery, passive range of motion exercises were begun. Active range of motion was initiated at 6 weeks postoperatively and strengthening exercises were begun at 12 weeks postoperatively, and these were continued until 5-6 months postoperatively.

\section{Functional Outcome Evaluation}

All patients in the study completed postoperative shoulder specific PROMs, including the American Shoulder and Elbow Surgeon's score (ASES), the Single Assessment Numerical Evaluation score (SANE), and the Constant Murley score (CS) at a minimum of 1 year or up to 2 years after surgery. To quantify the clinical significance of outcome achievement on the ASES, SANE, and CS scores, the principles of the MCID were applied as defined for functional PROMs. Prior work has proposed that the MCID be considered a minimum target for outcome improvement. ${ }^{15}$ Patients that reached or surpassed this previously established target were considered to have attained the MCID either on individual questionnaires or on any one questionnaire ("any MCID").

\section{Assessment of Geographical Location}

The institution where this study was conducted is located in a large metropolitan area with an approximate 50-mile cutoff between urban and suburban areas. Thus, patients within 50 miles of the institution were considered to be able to have ample transportation options for travel to postoperative follow-up visits and have access to physical therapists that were most familiar with the rehabilitation protocols used by the operating surgeons. Google Maps application programming interface was used to systematically fetch travel distances between patient's home postal codes and the specific institution's surgery center/clinical offices locations. Patients with home residences less than 50 miles from the institution were labeled as the local group, whereas those with home residence 50 miles or 
greater from the institution were labeled as the referral group. Published statistics from the U.S. Internal Revenue Service were used to determine the average adjusted gross income (AGI) to determine whether income was an effect modifier on the association between geographic location and achieving the MCID. The tax return information for the year 2017 was used for all patients, as it was the most recently published data.

\section{Statistical Analysis}

The relative density of patients in relation to the institution of study was summarized as a heatmap distribution. The geographical distribution of patients attaining MCID for each PROM (ASES, CS, and SANE) was summarized as geographical scatterplots, with marker size corresponding to the number of patients within each geographical area. Descriptive statistics for all continuous variables are reported as means and standard deviations, while categorical variables are reported as frequency and percentages. Where appropriate, differences between local and referral groups were assessed using independent $t$-tests or MannWhitney $U$-test for continuous data and $\chi^{2}$ tests of independence for categorical data. Postoperative differences in PROMs were assessed via paired-sample $t$-tests. Subgroup analysis of tear complexity was performed. Logistic regression of achieving any MCID was conducted, incorporating sex, age, body mass index (BMI), tear chronicity, tear complexity, AGI, primary insurance, and travel distance into the model. Continuous variables were recoded into categorical variables to facilitate interpretable results that are more readily translatable to clinical application. The Hosmer Lemeshow goodness-of-fit test was used to verify that the model was not improperly specified. The Pearson goodness-of-fit statistic was computed to quantify the similarity of quantities for each grouping yielded by the model. This statistic follows a $\chi^{2}$ distribution. A low $P$ value, implying dissimilarities in the groupings, indicates a poor fit.

\section{Results}

A total of 385 patients satisfied the inclusion criteria and were included in the final analysis. The mean BMI of the cohort was $31.08 \pm 7.12$, and the mean age was $60.0 \pm 8.99$ years. A majority of the cohort $(59 \%)$ was male. The mean driving distance from the surgical center was $34.13 \pm 39.75$ miles, with $83.9 \%$ of the cohort having a driving distance of $<50$ miles at the time of surgery. Figure 1 demonstrates a geospatial representation of density of patients included in this cohort in relation to the medical center where the procedures were performed. The local group traveled an average of $22.8 \pm 13.2$ miles, and the referral group traveled an average of $93.2 \pm 69.3$ miles to their orthopedic provider. There were no statistically significant

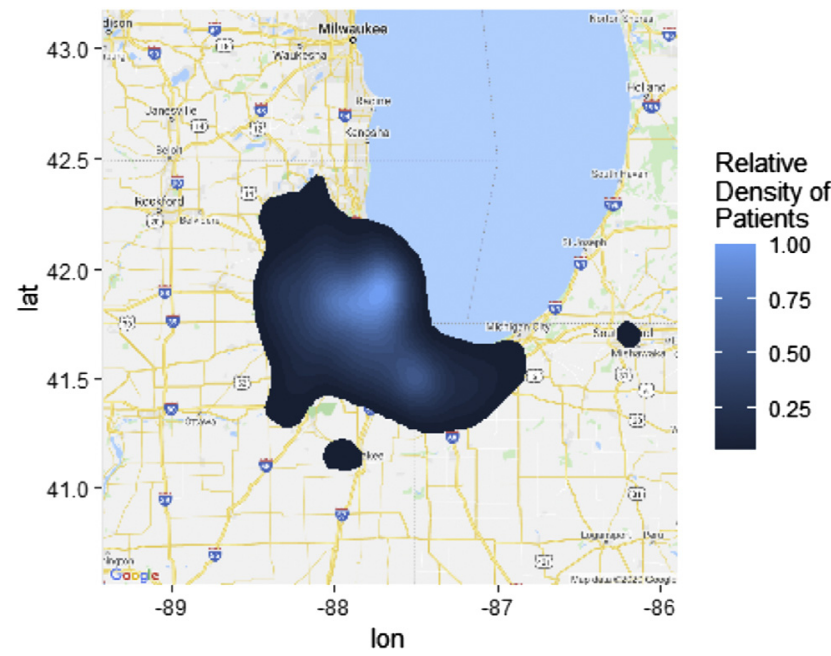

Fig 1. Geospatial representation of the relative density of patients included in this study relative to the high-volume referral center where the study took place.

differences between age, sex, BMI, symptom duration, or tear complexity between the local and referral groups (Table 1). A comparison of payor status demonstrated a significantly higher proportion of Medicare patients in the local group $(P=.011)$, while there was no difference in private insurance or Workman's Compensation. Comparative analysis of AGI revealed that patients in the local group lived in geographic areas with significantly higher incomes than those in the referral group $(65.6 \pm 49.2$ vs $43.1 \pm 35.1$ per US $\$ 1000$, respectively; $P<.001$ ) (Table 1).

\section{Comparison of Preoperative and Postoperative Outcome Scores}

The paired $t$-test of preoperative and postoperative functional scores revealed significant improvement in all outcomes scores over the 1-year period in all patients $(48.7 \pm 20.4$ preoperatively vs $83.8 \pm 18.5$ postoperatively for ASES, $13.9 \pm 7.1$ vs $24.6 \pm 8.3$ for Constant, $37.7 \pm 23$ vs $77.5 \pm 23.9$ for SANE; $P<.001$ for all). A comparison of preoperative and postoperative scores between the local and referral groups showed no statistically significant difference at either timepoint $(P>.05$ for all).

\section{Analysis of Achieving the MCID}

The ASES, Constant, and SANE threshold scores for achieving the MCID were $11.1,4.6$, and 16.9, respectively. Table 2 summarizes the numbers of patients in each group achieving the MCID. There was no statistically significant difference between the groups in the proportion of patients achieving the MCID for any outcome measure $(P>.05$ for all). A total of 305 patients $(94.4 \%)$ in the local group and 56 patients $(90.3 \%)$ in the referral group achieved the MCID for at least one PROM. The $\chi^{2}$ analysis demonstrated that 
Table 1. Patient Demographics

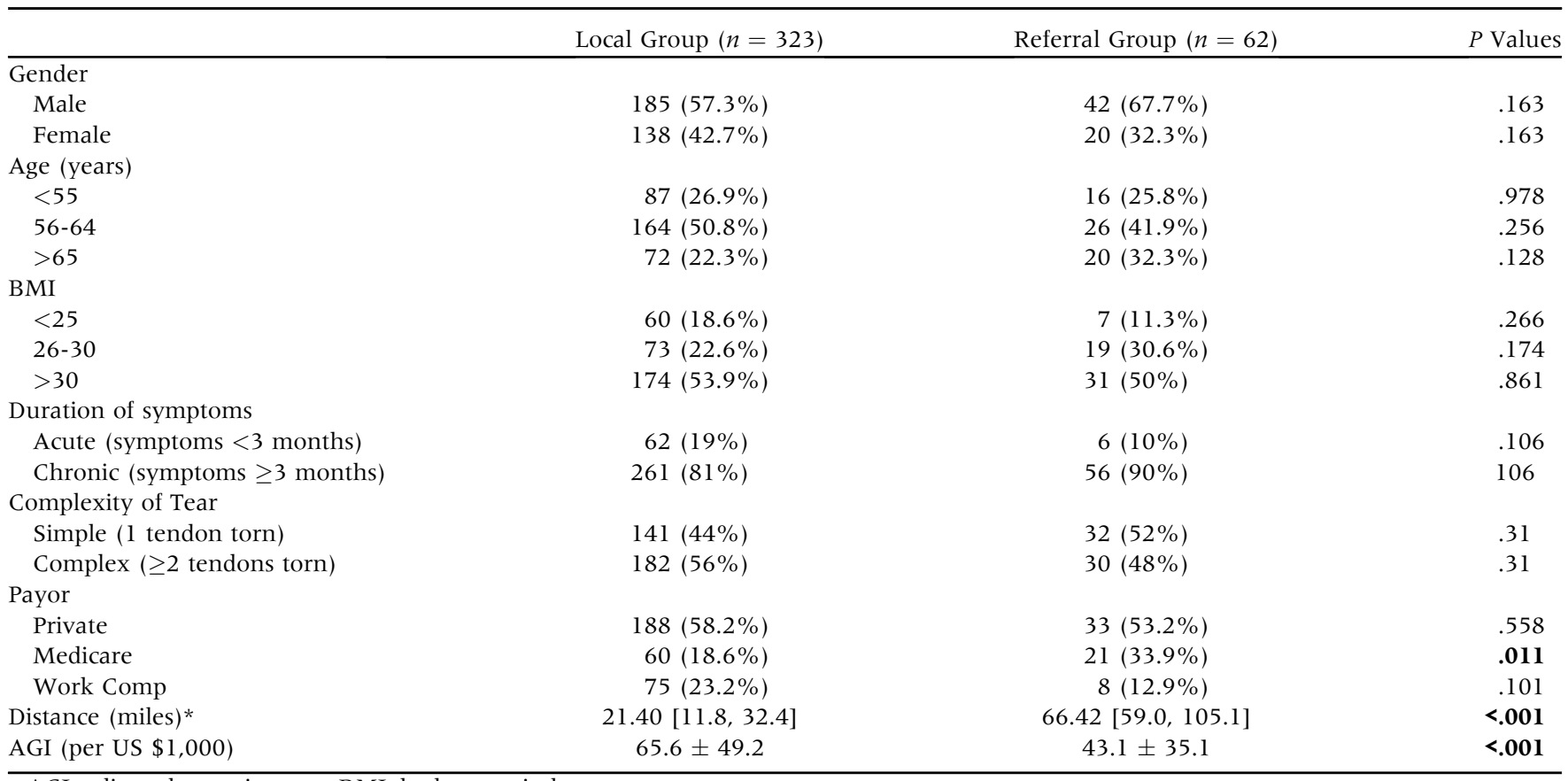

AGI, adjusted gross income; BMI, body mass index.

there was no statistically significant difference in rates of achieving the MCID between the two groups ( $P=$ .628). Figure 2 depicts representations of geographic densities of patients who achieved MCID on the various PROMs studied.

\section{Analysis of Achieving the MCID by Tear Complexity}

Subgroup analysis by tear complexity is outlined in Table 3. Briefly, there was no statistically significant difference in improvement from preoperative outcomes scores between local and referral groups in those with simple tears ( 1 tendon) or those with complex tears $(\geq 2$ tendons) $(P>.05$ for all). Among patients with complex tears, those in the referral group had significantly higher Constant scores at 1-year postoperatively than those in the local group $(P=.008)$. However, there was no statistically significant difference between rates of patients achieving the Constant score MCID within this subgroup $(P>.05)$. Similarly, there was no statistically significant difference in other postoperative outcomes scores within the tear complexity subgroups, nor was there a difference in rates of achieving the MCID $(P>$ .05 for all) (Table 3).

\section{Logistic Regression}

Table 4 summarizes the results of the logistic regression. The Pearson goodness-of-fit statistic yielded a $P$ value of 1 , indicating that the model had a good fit. Briefly, there was no significant difference between referral groups in achieving the MCID for any PROM when controlling for age, sex, adjusted gross income, primary health insurance, tear chronicity, and tear complexity. Although not statistically significant, female gender, older age, BMI $>30$, AGI $>\$ 150,000$, worker's compensation medical insurance, and travel distance $>50$ miles were all negatively associated with achievement of any MCID. On the other hand, nonobese BMI, chronic, or complex rotator cuff tear, and Medicare insurance all trended toward a mild positive association with any MCID achievement (Table 4).

\section{Discussion}

The primary finding of this study is that patients who underwent primary arthroscopic RCR that live in close

Table 2. Preoperative and l-Year Postoperative Functional Scores by Distance Group

\begin{tabular}{lccc}
\hline & Local Group & Referral Group & $P$ Values \\
\hline Preoperative & & & \\
$\quad$ ASES & $46.1 \pm 20.7$ & $49.1 \pm 20.4$ & .33 \\
Constant & $13.5 \pm 6.4$ & $14 \pm 7.2$ & .572 \\
$\quad$ SANE & $35.5 \pm 23.5$ & $38.1 \pm 22.9$ & .458 \\
Postoperative & & & \\
$\quad$ ASES & $82.2 \pm 19.2$ & $84.1 \pm 18.4$ & .491 \\
ASES MCID & $268(86.7 \%)$ & $47(83.9 \%)$ & .687 \\
Constant & $25.9 \pm 7$ & $24.4 \pm 8.5$ & .159 \\
Constant MCID & $211(82.4 \%)$ & $36(76.6 \%)$ & .452 \\
SANE & $76.7 \pm 27.1$ & $77.7 \pm 23.4$ & .801 \\
SANE MCID & $241(79.0 \%)$ & $45(78.9 \%)$ & 1 \\
Any MCID & $305(94.4 \%)$ & $56(90.3 \%)$ & .628 \\
\hline
\end{tabular}

ASES, American Shoulder and Elbow Score; MCID, minimal clinically important difference; SANE, Single Assessment Numeric Evaluation. 

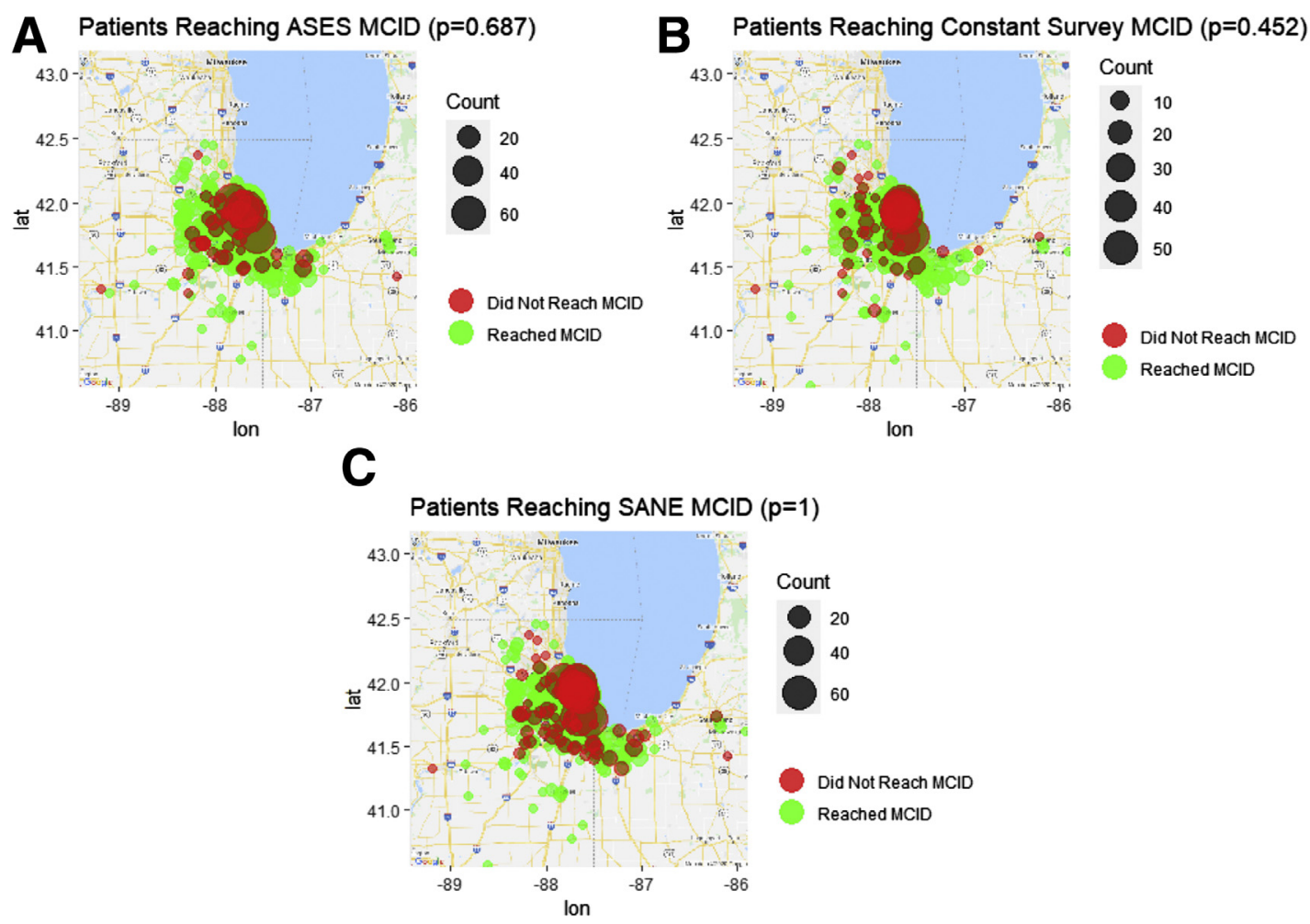

Fig 2. Geospatial representation of the relative density of patients achieving MCID for ASES Score (A), Constant Survey (B) and SANE Score $(\mathrm{C})$.

proximity to the tertiary referral center where the surgery took place had similar 1-year postoperative outcomes and similar rates of achieving the MCID as patients who travelled farther to undergo the procedure. There was no statistically significant difference in preoperative or postoperative PROMs between those who traveled $\geq 50$ miles to their orthopedic provider and those who traveled less. These findings suggest that travel distance does not significantly influence outcomes after arthroscopic rotator cuff repair.

Regionalization of elective surgery has been considered by health policy stakeholders as a strategy to maximize the quality and value of these procedures. ${ }^{16}$
With the establishment of COEs, there has been a recent focus in the literature regarding whether undergoing orthopedic procedures at high-volume centers affects outcomes. Laucis et al. used data from the National Inpatient Sample to demonstrate a trend of patients seeking arthroplasty procedures in high-volume hospitals from 2000 to 2012, and they showed that this manifested in lower complication rates. ${ }^{17}$ In an administrative study investigating over 2.5 million primary total hip or knee arthroplasty procedures, Dy et al. found that complication risk was higher if patients sought care at a local low-volume hospital versus a high-volume alternative. ${ }^{16}$ This effect has been

Table 3. Preoperative and 1-Year Postoperative Functional Scores by Distance and Tear Complexity

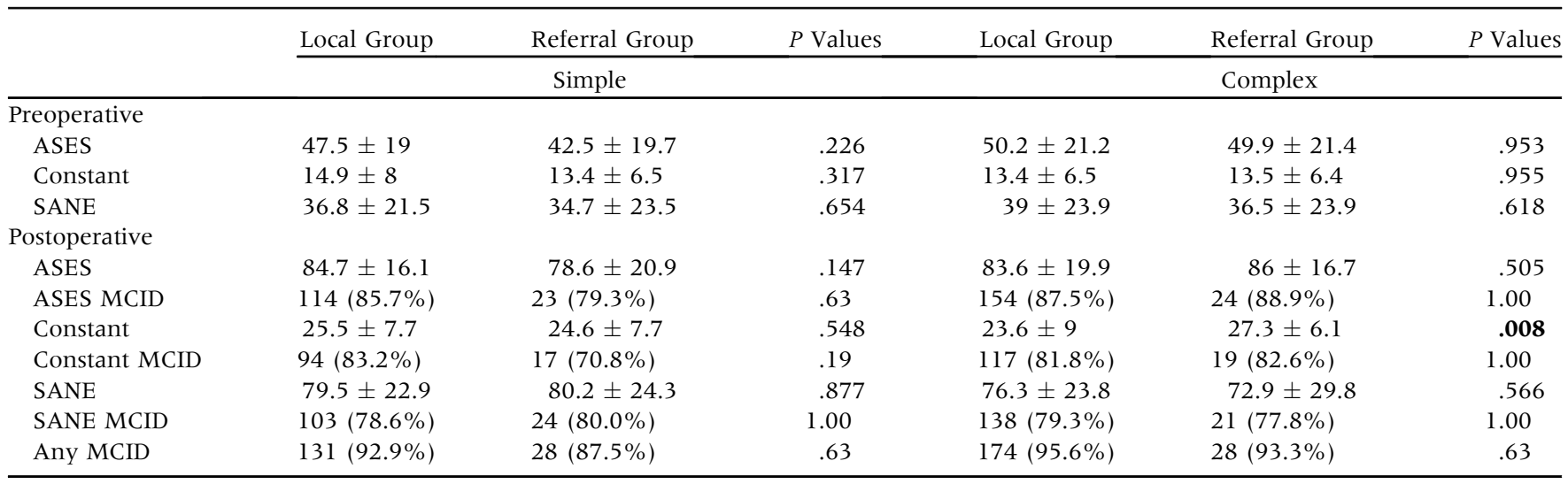


Table 4. Logistic Regression Analysis of Variables for Achieving Any MCID

\begin{tabular}{|c|c|c|c|c|}
\hline & OR & $2.5 \% \mathrm{CI}$ & $97.5 \% \mathrm{CI}$ & $P$ Values \\
\hline \multicolumn{5}{|l|}{ Sex } \\
\hline Male & Ref. & & & \\
\hline Female & .99 & .76 & 1.29 & .964 \\
\hline \multicolumn{5}{|l|}{ Age (years) } \\
\hline$<56$ & Ref. & & & \\
\hline $56-64$ & 1.00 & .75 & 1.34 & .998 \\
\hline$>64$ & .96 & .60 & 1.51 & .862 \\
\hline \multicolumn{5}{|l|}{ BMI } \\
\hline$<26$ & Ref. & & & \\
\hline $26-30$ & 1.02 & .69 & 1.52 & .907 \\
\hline$>30$ & 1.00 & .72 & 1.40 & .985 \\
\hline \multicolumn{5}{|l|}{ Chronicity } \\
\hline Acute (<3 months) & Ref. & & & \\
\hline Chronic & 1.00 & .74 & 1.39 & .985 \\
\hline \multicolumn{5}{|l|}{ Tear Type } \\
\hline Simple ( $<2$ tendons) & Ref. & & & \\
\hline Complex & 1.03 & .80 & 1.31 & .838 \\
\hline \multicolumn{5}{|l|}{ Adjusted gross income } \\
\hline$<\$ 75,000$ & Ref. & & & \\
\hline$\$ 75,000-149,000$ & 1.02 & .76 & 1.35 & .887 \\
\hline$>\$ 150,000$ & .99 & .62 & 1.50 & .958 \\
\hline \multicolumn{5}{|l|}{ Primary insurance } \\
\hline Private & Ref. & & & \\
\hline Medicare & 1.01 & .65 & 1.57 & .957 \\
\hline Worker's compensation & .94 & .67 & 1.30 & .723 \\
\hline Driving distance $>49$ miles & .94 & .66 & 1.33 & .752 \\
\hline
\end{tabular}

demonstrated in the field of shoulder surgery as well. ${ }^{18,19}$ Weinheimer et al. conducted a systematic review and meta-analysis that showed increased complications, length of stay, surgical time, and surgical cost in shoulder arthroplasty and RCR when performed by a low-volume shoulder surgeon, defined as $<5$ arthroplasties and/or $<12$ rotator cuff repairs annually. ${ }^{7}$ Using a large national database, Ramkumar et al. found that that length of stay and cost after total shoulder arthroplasty was inversely correlated with hospital and surgeon volume. ${ }^{20}$

A by-product of concentrating high-volume providers in COEs is that patients may have to travel longer distances to receive care. A "travel distance bias" has been described in the oncologic literature, which suggests that patients traveling longer distances are healthier and suffer fewer complications. ${ }^{21,22}$ However, the opposite effect ("reverse travel distance bias") has been demonstrated in the general surgery literature, with patients who travel longer distances suffering increased complications. ${ }^{8}$ For patients who receive care away from their immediate geographic area, established medical providers may not be readily accessible upon returning home, making it challenging to identify and manage complications. Few studies have examined this effect in the orthopedic literature. Using an institutional database at a center of excellence for total joint arthroplasty, Nwachukwu et al. demonstrated no significant association between patient travel distance and complication risk. ${ }^{10}$ Furthermore, Beck et al. showed that travel distance of $\geq 50$ miles from a specialty center had no effect on postoperative outcomes or achieving the MCID 2 years after undergoing hip arthroscopy for femoroacteabular impingement syndrome. ${ }^{9}$

Given the established volume-outcomes relationship in arthroscopic RCR and other similar procedures, the existence of a reverse travel distance bias could nullify the purported benefits of COEs. Although the current study did not directly compare outcomes at COEs versus other facilities, our results corroborate the lack of a reverse travel distance bias for orthopedic procedures found in prior studies, supporting the potential for COEs to fulfill their intended purpose. Our results set the stage for future studies to investigate the effectiveness of COEs and the comparative outcomes of procedures performed in these facilities versus others.

Multiple tendon involvement has been associated with less favorable outcomes after rotator cuff repair. ${ }^{23-25}$ However, our subgroup analysis showed no effect of tear complexity on achieving the MCID on any PROM between referral groups at minimum 1 year postoperatively. These results suggest that patients with more complex tear patterns may achieve comparable functional outcomes to those with simpler tears when seeking care at a high-volume center despite traveling farther for care. Furthermore, our data suggest that patients with more severe pathology who seek out higher-volume surgeons away from their immediate geographic area may be more motivated or may have better support during their recovery, setting them up for an improved outcome.

Future studies should investigate the associated economic impact of a greater travel distance, particularly the costs incurred because of factors such as travel and time off work. The local group had a significantly higher AGI in our cohort, potentially due to the demographics of the surrounding area to the study location. However, it is possible that those travelling a farther distance may be already at an economic disadvantage, which will be exacerbated by the costs of traveling to a COE. The present study lays the groundwork for this effect to be studied on a larger scale across various geographic areas.

\section{Limitations}

This study has a number of limitations to note. First, this cohort consisted of patients who were operated on by multiple providers from the same institution, which might introduce minor variability in surgical technique and postoperative rehabilitation protocols. However, all surgeons who perform a high volume of RCR procedures were part of the same institution and had similar surgical techniques and postoperative rehabilitation protocols. Second, our use of a 50-mile cutoff is not based on a previously established distance but rather 
was chosen on the basis of the radius from our particular metropolitan area and its use in prior similar studies. ${ }^{9}$ Third, this study was conducted in a large metropolitan area and may not be generalizable to other geographic locations. Finally, this study does not compare outcomes of RCR at high-volume versus lowvolume centers, or at COEs versus other facilities.

\section{Conclusion}

When controlling for age, sex, AGI, primary health insurance, tear chronicity, and tear complexity, distance to a high-volume shoulder arthroscopy surgery center did not have an effect on achieving the MCID for any PROM at least 1 year after arthroscopic rotator cuff repair.

\section{References}

1. Bozic KJ, Maselli J, Pekow PS, Lindenauer PK, Vail TP, Auerbach AD. The influence of procedure volumes and standardization of care on quality and efficiency in total joint replacement surgery. J Bone Joint Surg Am 2010;92: 2643-2652.

2. Malik AT, Jain N, Scharschmidt TJ, Li M, Glassman AH, Khan SN. Does surgeon volume affect outcomes following primary total hip arthroplasty? A systematic review. J Arthroplasty 2018;33:3329-3342.

3. Wilson S, Marx RG, Pan T-J, Lyman S. Meaningful thresholds for the volume-outcome relationship in total knee arthroplasty. JBJS 2016;98:1683-1690.

4. Basques BA, Bitterman A, Campbell KJ, Haughom BD, Lin J, Lee S. Influence of surgeon volume on inpatient complications, cost, and length of stay following total ankle arthroplasty. Foot Ankle Int 2016;37:1046-1051.

5. Mehta N, Chamberlin P, Marx RG, et al. Defining the learning curve for hip arthroscopy: A threshold analysis of the volume-outcomes relationship. Am J Sports Med 2018;46:1284-1293.

6. Cole T, Veeravagu A, Zhang M, Ratliff JK. Surgeon procedure volume and complication rates in anterior cervical discectomy and fusions. Clin Spine Surg 2017;30:E633-E639.

7. Weinheimer KT, Smuin DM, Dhawan A. Patient outcomes as a function of shoulder surgeon volume: A systematic review. Arthroscopy 2017;33:1273-1281.

8. Etzioni DA, Fowl RJ, Wasif N, Donohue JH, Cima RR. Distance bias and surgical outcomes. Med Care 2013;51: 238-244.

9. Beck EC, Nwachukwu BU, Lee EK, et al. Travel distance does not affect outcomes in hip preservation surgery: A case for centers of excellence. Orthop J Sports Med 2020;8: 2325967120908821.

10. Nwachukwu BU, Dy CJ, Burket JC, Padgett DE, Lyman S. Risk for complication after total joint arthroplasty at a center of excellence: The impact of patient travel distance. J Arthroplasty 2015;30:1058-1061.
11. Ensor KL, Kwon YW, Dibeneditto MR, Zuckerman JD, Rokito AS. The rising incidence of rotator cuff repairs. J Shoulder Elbow Surg 2013;22:1628-1632.

12. Gowd AK, Liu JN, Garcia GH, Cabarcas BC, Verma NN. Arthroscopic massive rotator cuff repair and techniques for mobilization. Arthrosc Tech 2018;7:e633-e638.

13. Cole BJ, El Attrache NS, Anbari A. Arthroscopic rotator cuff repairs: An anatomic and biomechanical rationale for different suture-anchor repair configurations. Arthroscopy 2007;23:662-669.

14. Abrams GD, Gupta AK, Hussey KE, et al. Arthroscopic repair of full-thickness rotator cuff tears with and without acromioplasty: Randomized prospective trial with 2-year follow-up. Am J Sports Med 2014;6:1296-1303.

15. Cvetanovich GL, Gowd AK, Liu JN, et al. Establishing clinically significant outcome after arthroscopic rotator cuff repair. J Shoulder Elbow Surg 2019;28:939-948.

16. Dy CJ, Marx RG, Ghomrawi HM, Pan TJ, Westrich GH, Lyman S. The potential influence of regionalization strategies on delivery of care for elective total joint arthroplasty. J Arthroplasty 2015;30:1-6.

17. Laucis NC, Chowdhury M, Dasgupta A, Bhattacharyya T. Trend toward high-volume hospitals and the influence on complications in knee and hip arthroplasty. J Bone Joint Surg Am 2016;98:707-712.

18. Hammond JW, Queale WS, Kim TK, McFarland EG. Surgeon experience and clinical and economic outcomes for shoulder arthroplasty. J Bone Joint Surg Am 2003;85: 2318-2324.

19. Jain N, Pietrobon R, Hocker S, Guller U, Shankar A, Higgins LD. The relationship between surgeon and hospital volume and outcomes for shoulder arthroplasty. J Bone Joint Surg Am 2004;86:496-505.

20. Ramkumar PN, Navarro SM, Haeberle HS, Ricchetti ET, Iannotti JP. Evidence-based thresholds for the volumevalue relationship in shoulder arthroplasty: Outcomes and economies of scale. J Shoulder Elbow Surg 2017;26: 1399-1406.

21. Lamont EB, Hayreh D, Pickett KE, et al. Is patient travel distance associated with survival on phase II clinical trials in oncology? J Natl Cancer Inst 2003;95:1370-1375.

22. Lenhard RE, Enterline JP, Crowley J, Ho GY. The effects of distance from primary treatment centers on survival among patients with multiple myeloma. J Clin Oncol 1987;5:1640-1645.

23. Novoa-Boldo A, Gulotta LV. Expectations following rotator cuff surgery. Curr Rev Musculoskelet Med 2018;11: 162-166.

24. Lambers Heerspink FO, Dorrestijn O, van Raay JJ, Diercks RL. Specific patient-related prognostic factors for rotator cuff repair: A systematic review. J Shoulder Elbow Surg 2014;23:1073-1080.

25. Nho SJ, Brown BS, Lyman S, Adler RS, Altchek DW, MacGillivray JD. Prospective analysis of arthroscopic rotator cuff repair: Prognostic factors affecting clinical and ultrasound outcome. J Shoulder Elbow Surg 2009;18: 13-20. 